\section{Likelihood Ratio, negatives}

R.-D. Hilgers ${ }^{1}$, N. Heussen ${ }^{1}$ und S. Stanzel ${ }^{2}$

${ }^{1}$ Institut für Medizinische Statistik, Universitätsklinikum der RWTH Aachen, Aachen, Deutschland

${ }^{2}$ Heidelberg, Deutschland

Synonym(e) Likelihood Ratio für ein negatives Testergebnis; Likelihood-Quotient; LR-

Englischer Begriff negative likelihood ratio
Definition Das negative Likelihood Ratio ist definiert als der Quotient von (1-Sensitivität) und Spezifität ( $\triangleright$ Sensitivität, diagnostische; $\triangleright$ Spezifität, diagnostische).

\section{Literatur}

Hilgers R-D, Bauer P, Scheiber V (2002) Einführung in die Medizinische Statistik. Springer, Berlin/Heidelberg/New York 